\title{
DRIVING WITH FORESIGHT - EVALUATING THE EFFECT OF COGNITIVE DISTRACTION AND EXPERIENCE ON ANTICIPATING EVENTS IN TRAFFIC
}

\author{
Kristin Mühl ${ }^{1}$, Valentin Koob ${ }^{2}$, Tanja Stoll ${ }^{1}$ \& Martin Baumann ${ }^{1}$ \\ ${ }^{1}$ Ulm University, Germany \\ ${ }^{2}$ Technische Universität Berlin, Germany \\ Email: kristin.muehl@uni-ulm.de
}

\begin{abstract}
Summary: Driving with foresight is essential for road safety. Anticipating upcoming events and intended maneuvers of other traffic participants requires the perception and processing of meaningful and valid cues. To provide insights into the cognitive mechanisms of anticipation, we investigated the effect of cognitive load, experience and cue characteristic on the anticipation of upcoming lane changes in urban driving scenarios. A two-step reaction method gathered low and high certainty anticipatory reactions of student and ambulance drivers. Results indicated that different anticipatory cues affected anticipatory performance. Target cues highly associated with the intended behavior of another traffic participant increased while context cues in the surrounding environment seemed to hamper anticipatory reactions. Furthermore, high cognitive load prolonged the latencies of low certainty anticipation but did not affect the performance quality. This initial intuition of an upcoming lane change was indicated earlier by experienced than by inexperienced drivers. These findings enhance the understanding of the human process of anticipation in dynamic uncritical traffic situations.
\end{abstract}

\section{INTRODUCTION}

Safe Driving is a matter of anticipatory driving. Drivers need to be aware of the situation to perform adequately. Following the theory of situational awareness, it requires the perception of relevant situational elements as well as the integration and comprehension of this information to predict the intended behavior of other road users (Endsley, 1995). People are able to anticipate while driving based on gained knowledge about stereotypical traffic situations (Stahl, Donmez, \& Jamieson, 2014). Because experienced drivers encountered a large number of traffic situations, they perform better in anticipation and hazard perception tasks. More specifically, they are faster in anticipating hazards (Patten, Kircher, Östlund, Nilsson, \& Svenson, 2006) and can predict more hazards correctly than inexperienced drivers (Jackson, Chapman, \& Crundall, 2009).

Anticipation is known as a high-level cognitive competence (Stahl et al., 2014), which is deteriorated by cognitive load due to inferences with central executive functions (Baumann, Petzoldt, Groenewoud, Hogema, \& Krems, 2008; Mühl \& Baumann, 2018). Even if the relevant information has been perceived before, induced cognitive load inhibits the integration of this information in the situation model (Baumann et al., 2008) and therefore reduces anticipation. While driving in real traffic, novice drivers were observed to have smaller workload reservecapacity compared to experienced drivers (Patten et al., 2006), as fewer processes of the driving task follow automatic patterns. This finding might explain why young drivers have increased crash involvement probabilities (Deery, 1999). 
Furthermore, anticipation is determined by the perception of relevant characteristic cues (Stahl et al., 2014). We follow the proposed classification of characteristic cues by Mühl, Stoll, and Baumann (2019). They defined causal cues, which contain a reason for a potential change in the upcoming behavior, as the basis for anticipation. These can be, for instance, another car on the adjacent lane approaching a blockage on the road which enables the prediction of a lane change. Moreover, drivers can benefit from additional situational characteristics in the environment. Mühl et al. (2019) differentiated between target and context cues. Elements triggering the intention of another's future behavior directly by the perception of a single cue are called target cues. For example, an indicator is strongly linked to lane change behavior in a specific direction. By contrast, context cues do not provide information about others' intentions but can attract and focus attention to a specific event (e.g., like a construction site traffic sign). In a study from Mühl et al. (2019), anticipatory performance increased and was less affected by cognitive load, when a target cue was visible compared to the presence of a context cue. However, as most of the participants were inexperienced drivers, we aimed to conduct a similar study with a highly experienced sample. We assumed inexperienced drivers to benefit more from the presence of additional cues than experienced drivers, especially when being highly cognitively demanded. This expectation is based on the findings of reduced workload reserve-capacity (Patten et al., 2006) and decreased pre-knowledge of inexperienced drivers, that hampers the prediction of future scenarios due to the insufficient situation model (Durso, Rawson, \& Girotto, 2007). Additional cues might easily extend the situation model with information that was already available for experienced drivers.

The study aims to investigate the effects of cognitive distraction and experience on the processing of anticipatory cues. We decided to test highly experienced drivers (paramedics), who are used to drive ambulances in stressful and difficult situations. Given their daily need to anticipate other traffic participants’ behavior, we assumed them to have developed higher anticipatory skills in traffic compared to student drivers with low annual mileage.

\section{METHOD}

\section{Participants}

The driver sample consisted of 11 experienced and 26 inexperienced young drivers. Experienced (ambulance) drivers were recruited by announcements at emergency departments whereas inexperienced drivers were recruited from the student body of the Ulm University. The largest differences in these subgroups could be identified for gender and annual driving distance (see table 1 for an overview of sample characteristics). Experienced drivers drove at least 50000 $\mathrm{km} /$ year whereas inexperienced drivers did not exceed $10000 \mathrm{~km} /$ year.

Table 1. Sample characteristics for the subgroups of experience

\begin{tabular}{|c|c|c|}
\hline & experienced drivers & inexperienced drivers \\
\hline age in years & $M=25.4(S D=1.14)$ & $M=21.9(S D=0.46)$ \\
\hline $\operatorname{sex}$ & $\widehat{o} \mathrm{n}=9, \stackrel{+}{\mathrm{n}}=2$ & $\widehat{\partial} \mathrm{n}=7, q \mathrm{n}=19$ \\
\hline \multicolumn{3}{|l|}{ education } \\
\hline school-leaving qualification & $\mathrm{n}=3$ & $\mathrm{n}=0$ \\
\hline general qualification for university entrance & $\mathrm{n}=7$ & $\mathrm{n}=18$ \\
\hline university degree & $\mathrm{n}=1$ & $\mathrm{n}=8$ \\
\hline \multicolumn{3}{|l|}{ driving experience } \\
\hline possession of driving license in years & $M=7.9(S D=1.01)$ & $M=4.2(S D=0.52)$ \\
\hline annual driving distance in kilometer/year & $M=56090(S D=4442)$ & $M=4588(S D=1522)$ \\
\hline
\end{tabular}




\section{Design \& Procedure}

We conducted a video-based laboratory experiment using urban lane change scenarios from a driver's perspective. A two-step reaction paradigm was performed in which participants had to state the point in time of the initial intuition (low certainty anticipation) and the high confidence (high certainty anticipation) by button press that another car might enter the own lane. A 2 x 2 x $2 \times 2$ mixed design was applied to test the hypotheses. Cognitive load (low vs high), target cues (indicator vs no indicator), and context cues (traffic sign or no traffic sign) were varied using a within-subjects design. Experience was a between-group variable.

Overall, the experiment lasted about 60 minutes. Participants watched 24 randomized videos comprising 12 stimulus videos (three repetitions of each cue combinations using different scenarios, see the next section for more details) and 12 distractor videos within each trial. Stimulus videos contained anticipatory cues showing a reason for a lane change while distractor videos did not. This balance was chosen to ensure participants' attention because they were only able to anticipate in half of the trials. The participants encountered two trials, one with a low cognitive demanding (=low load) and one with a high cognitive demanding (=high load) secondary task. The additional secondary tasks were presented in a blocked design and random order. At the beginning of each trial, people had three practice scenarios to get familiar with the secondary task. At the end of the experiment, they completed a demographic questionnaire and were rewarded for participation.

\section{Material}

The experiment was set up using the software PsychoPy. Videos of urban two-lane road scenarios were developed lasting between 24 and 40 seconds. Participants saw another car on the adjacent lane approaching an obstacle (a bus, parked cars or a construction site) from a driver's perspective in the stimulus videos. An indicator was used as the target cue starting with a time to collision (TTC) of 5 seconds before the video ended, which matched the time when the car would initiate a lane change. For the context cue, a traffic sign was placed $150 \mathrm{~m}$ in front of the blockage (TTC was 18 seconds) which was related to the kind of obstacle. The videos ended with a TTC of 4 seconds between the ego vehicle and the observed car, which also had a TTC of 4 seconds towards the blockade. Distractor videos were comparable regarding length and situational elements but did not include any obstacle.

While watching these videos, participants listened to four letters presented with an inter-stimulus interval of $2000 \mathrm{~ms}$ in an alternate order: "P”, “W”, “D”, "G”. In the low cognitive load condition, participants had to recall the letter " $D$ " as soon as they heard this letter while watching 24 scenarios. The high cognitive load condition comprised a 2-back memory updating task.

\section{Data analyses}

To investigate the effects of workload, cue characteristic, and experience a two-level mixed model approach was used to address the repeated measurements as well as the unequal number of observations per participant. Given the binary nature of the anticipatory reactions (button press yes or no), a multilevel logistic regression analysis (via generalized linear mixed models, GLMM) were fitted using a logit link function. Anticipatory latencies (in seconds) describe the time that passed between the onset of a primary cue and the participant's reaction. They were 
analyzed using linear mixed models (LME) assuming a normal distribution of errors and using an identity link function. Each model was built up in the same manner. First, a random intercept model without any fixed effects was set up to calculate the interclass correlation (ICC) using participants' ID as a grouping variable. Then, workload, cue characteristic, and experience as categorical fixed effects were added. Note, that categorical variables were effect coded. Models were fitted using the lme4 package version 1.1-18-1 (Bates, Mächler, Bolker, \& Walker, 2015).

\section{RESULTS}

For the low and high certainty anticipatory reactions, 851 observations could be derived. Regarding low certainty anticipation, no differences were observed for the different cue conditions (see Fig. 1I). A missing target cue reduced the number of high certainty anticipatory reactions considerably (see Fig. 1II). Neither experience nor workload seemed to affect anticipatory response.
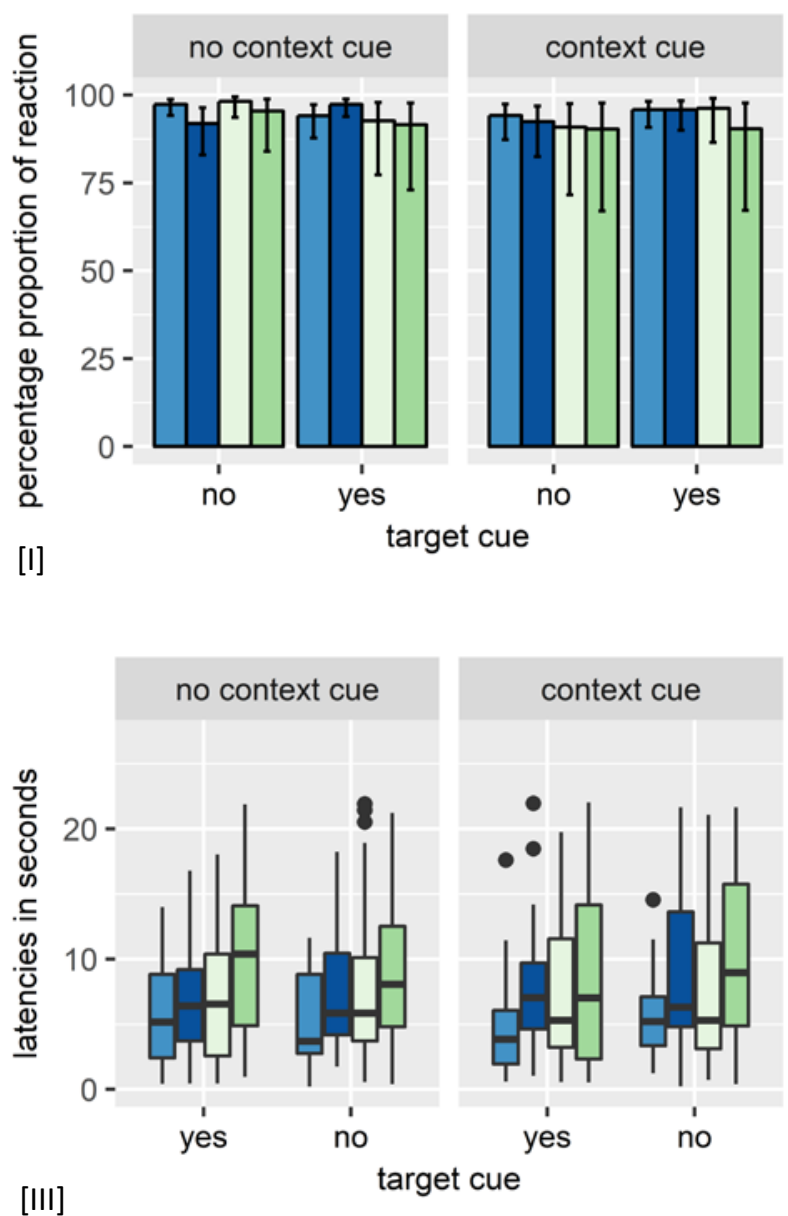

[III]

experienced low load experienced high load
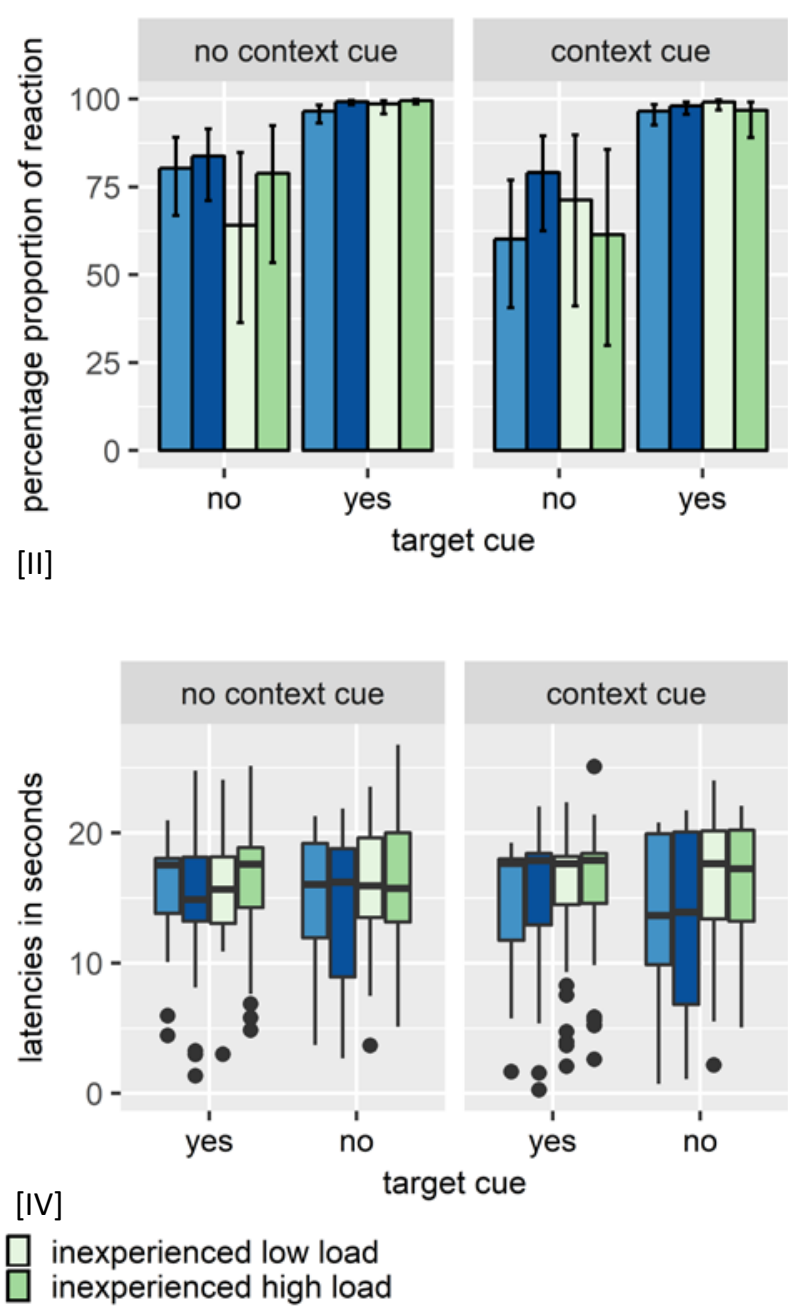

Figure 1. [I \& II] Percentage of anticipatory reactions (with 95\% confidence interval) and [III \& IV] boxplots of anticipatory latencies of [I \& III] low certainty anticipation and [II \& IV] high certainty anticipation affected by experience, cognitive load and cue characteristics (target and context cue) 
For inferential statistics of low and high anticipatory reactions, a mixed model approach was chosen given a substantial variability between the participants $\left(\right.$ ICC $_{\text {lowAnticipation }}=.476$, ICC $_{\text {highAnticipation }}=.306$ ). Results are displayed in Table 2. We observed a significantly higher chance for high certainty anticipation with a target cue $(p<.001)$. Furthermore, no context cue led to a marginally increased number of high certainty anticipatory reactions $(p=.087)$. This trend was also observable for low certainty anticipation indicated by the (close to significant) interaction of target and context cues $(p=.088)$ Overall, experience and cognitive load did not affect anticipatory reactions.

Table 2. Coefficients for the GLMM with workload, cue characteristic, and experience as predictors and anticipatory reaction as the criterion variable

\begin{tabular}{|c|c|c|c|c|c|c|c|c|c|c|}
\hline & \multicolumn{2}{|c|}{$b$} & \multicolumn{2}{|c|}{$S E$} & \multicolumn{2}{|c|}{$Z$} & \multicolumn{4}{|c|}{$p$} \\
\hline $\begin{array}{l}\text { Certainty of anticipatory } \\
\text { reaction }\end{array}$ & Low & High & Low & High & Low & High & Low & & High & \\
\hline Intercept & 2.865 & 2.574 & 0.406 & 0.357 & 7.053 & 7.201 & $<.001$ & $* * *$ & $<.001$ & $* * *$ \\
\hline Cognitive load (low) - CL & 0.174 & -0.213 & 0.136 & 0.157 & 1.272 & -1.357 & .203 & & .175 & \\
\hline Experience (high) - EH & 0.122 & -0.062 & 0.382 & 0.348 & 0.320 & -0.178 & .749 & & .858 & \\
\hline Target Cue (no) - TCN & -0.010 & -1.568 & 0.136 & 0.175 & -0.077 & -8.942 & .939 & & $<.001$ & $* * *$ \\
\hline Context Cue (no) - CCN & 0.170 & 0.268 & 0.136 & 0.157 & 1.246 & 1.713 & .213 & & .087 & $(*)$ \\
\hline $\mathrm{CL} * \mathrm{EH}$ & -0.097 & -0.188 & 0.136 & 0.157 & -0.709 & -1.197 & .478 & & .231 & \\
\hline $\mathrm{CL} * \mathrm{TCN}$ & 0.132 & 0.033 & 0.136 & 0.157 & 0.969 & 0.209 & .333 & & .835 & \\
\hline $\mathrm{EH} * \mathrm{TCN}$ & -0.157 & 0.251 & 0.136 & 0.172 & -1.156 & 1.462 & .248 & & .144 & \\
\hline $\mathrm{CL} * \mathrm{CCN}$ & 0.005 & -0.243 & 0.136 & 0.157 & 0.038 & -1.549 & .969 & & .121 & \\
\hline $\mathrm{EH} * \mathrm{CCN}$ & -0.070 & 0.005 & 0.136 & 0.156 & -0.512 & 0.034 & .608 & & .973 & \\
\hline $\mathrm{TCN} * \mathrm{CCN}$ & 0.233 & -0.041 & 0.137 & 0.156 & 1.709 & -0.260 & .088 & $(*)$ & .795 & \\
\hline $\mathrm{CL} * \mathrm{EH}^{*} \mathrm{TCN}$ & 0.148 & 0.081 & 0.136 & 0.157 & 1.085 & 0.520 & .278 & & .603 & \\
\hline $\mathrm{CL} * \mathrm{EH} * \mathrm{NCC}$ & 0.001 & 0.217 & 0.136 & 0.157 & 0.011 & 1.383 & .992 & & .167 & \\
\hline $\mathrm{CL} * \mathrm{TCN} * \mathrm{CCN}$ & 0.213 & 0.180 & 0.136 & 0.156 & 1.563 & 1.151 & .118 & & .250 & \\
\hline $\mathrm{EH} * \mathrm{TCN} * \mathrm{CCN}$ & -0.149 & 0.092 & 0.136 & 0.156 & -1.093 & 0.587 & .274 & & .557 & \\
\hline $\mathrm{CL} * \mathrm{EH} * \mathrm{TCN} * \mathrm{CCN}$ & -0.003 & 0.016 & 0.136 & 0.156 & -0.023 & 0.104 & .982 & & .917 & \\
\hline
\end{tabular}

Note. low certainty anticipatory performance: AIC $=544, \mathrm{BIC}=625$; high certainty anticipatory performance: AIC $=601$, $\mathrm{BIC}=681$; coefficients (b) of cognitive load, experience, target and context cue can be interpreted as the log odds of an anticipatory reaction relative to the grand mean, averaged over every other factor level; SE refers to the estimated standard error; $\mathrm{z}$ values were obtained using the Wald z-test; $\left({ }^{*}\right) p<.01 ;{ }^{*} p<.05$.; ${ }^{* *} p<.01 . ;{ }^{* * *} p<.001$

For low and high certainty anticipatory latencies, 707 and 693 observations could be derived. Low certainty anticipatory latencies seemed to be stronger affected by the independent variables than high certainty latencies (see Fig. 1 III \& IV). To test our assumptions, a mixed model approach was necessary due to the substantial variability between the participants $\left(\right.$ ICClowAnticipation $=.429$, ICC $\left._{\text {highAnticipation }}=.409\right)$. Results showed (see Table 3 ) that participants reacted about 1 second faster with low compared to high cognitive load for low certainty anticipation. More specifically, compared to the averaged reaction latencies of each independent value, experienced drivers with low cognitive load showed a faster low anticipation reaction when there was no cue at all. However, workload did not affect the latencies of high certainty anticipation. Experienced drivers were faster (about 1 second) than inexperienced driver by trend $(p=.097)$ for low certainty anticipation. Furthermore, experienced participants tended to react even faster in the high load condition. However, this tendency did not reach statistical significance $(p=.080)$. 
PROCEEDINGS of the Tenth International Driving Symposium on Human Factors in Driver Assessment, Training and Vehicle Design

Table 3. Coefficients for the LME with workload, cue characteristic, and experience as predictors and the reaction latencies of the anticipatory reactions as the criterion variable

\begin{tabular}{lrrrrrrrrr}
\hline & \multicolumn{2}{c}{$b$} & \multicolumn{2}{c}{ SE } & \multicolumn{2}{c}{$t$} & \multicolumn{2}{c}{$p$} \\
\hline $\begin{array}{l}\text { Certainty of anticipatory } \\
\text { reaction }\end{array}$ & \multicolumn{1}{c}{ Low } & \multicolumn{1}{c}{ High } & \multicolumn{1}{c}{ Low } & High & Low & High & Low & High \\
\hline Intercept & 7.515 & 15.758 & 34.186 & 35.779 & 11.883 & 28.454 & $<.001$ & $* * *$ & $<.001$ \\
Cognitive load (low) - CL & -1.000 & 0.188 & 661.457 & 643.874 & -6.154 & 1.234 & $<.001$ & $* * *$ & .218 \\
Experience (high) - EH & -1.078 & -0.665 & 34.186 & 35.779 & -1.705 & -1.202 & $.097\left(^{*}\right)$ & .237 \\
Target Cue (no) - TCN & 0.228 & 0.102 & 656.088 & 647.325 & 1.429 & 0.660 & .153 & .510 \\
Context Cue (no) - CCN & -0.020 & 0.086 & 656.896 & 643.19 & -0.127 & 0.566 & .899 & .572 \\
CL * EH & -0.074 & 0.267 & 661.457 & 643.874 & -0.452 & 1.752 & .651 & $.080\left(^{*}\right)$ \\
CL * TCN & -0.082 & 0.227 & 656.067 & 643.871 & -0.513 & 1.491 & .608 & .137 \\
EH * TCN & 0.109 & -0.130 & 656.088 & 647.325 & 0.680 & -0.838 & .497 & .402 \\
CL *CN & 0.128 & 0.101 & 656.518 & 643.319 & 0.797 & 0.663 & .426 & .507 \\
EH * CCN & -0.076 & 0.053 & 656.896 & 643.19 & -0.475 & 0.348 & .635 & .728 \\
TCN * CCN & -0.273 & 0.052 & 656.343 & 643.197 & -1.709 & 0.343 & $.088\left(^{*}\right)$ & .732 \\
CL * EH* TCN & -0.060 & 0.079 & 656.067 & 643.871 & -0.375 & 0.520 & .708 & .603 \\
CL * EH * NCC & 0.190 & 0.061 & 656.518 & 643.319 & 1.189 & 0.399 & .235 & .690 \\
CL * TCN * CCN & 0.107 & -0.125 & 656.312 & 643.458 & 0.671 & -0.821 & .503 & .412 \\
EH * TCN * CCN & 0.127 & 0.050 & 656.343 & 643.197 & 0.796 & 0.331 & .426 & .741 \\
CL *EH * TCN * CCN & -0.331 & -0.206 & 656.312 & 643.458 & -2.068 & -1.356 & $.039 *$ & .176 \\
\hline
\end{tabular}

Note. low certainty anticipatory performance: AIC = 4086, BIC = 4168; high certainty anticipatory performance: AIC = 3886, BIC = 3950; coefficients (b) of cognitive load, experience, target and context cue can be interpreted as the latencies of anticipatory reactions relative to the grand mean, averaged over every other factor level; SE refers to the estimated standard error; degrees of freedom were obtained using the Satterthwaite approximation; $\left({ }^{*}\right) p<.01$; ${ }^{*} p<.05 . ; * * p<.01 . ; * * p<.001$

\section{DISCUSSION}

The current study examined differences between inexperienced and experienced drivers in the anticipation of dynamic traffic situations regarding cognitive load and the availability of anticipatory cues. The applied two-step reaction method provides an extension presenting further insights into the cognitive process of anticipation (compared with the one-button reaction in hazard perception tests) since results differed for low and high certainty anticipation. For instance, high cognitive load increased latencies for about one second for the initial intuition of a lane change but did not affect the highly confident anticipatory reaction. Since the understanding of the situation precedes in cycles testing the prior developed assumptions (Durso et al., 2007), anticipation can be described as a process moderated by the subjective certainty about the occurrence of the predicted event.

As assumed, people highly benefitted from target cues, especially for the high certainty anticipation. Overall, highly experienced drivers were observed to be about one second faster in low certainty reactions. This finding did not reach significance, which might be a consequence of the small sample size. The samples differed with regard to the proportion of gender, but gender is unlikely to account for differences between the subsamples since we focused on cognitive processes of anticipation. Related studies showed that female and male drivers did not differ in their hazard perception skills (Scrimgeour, Szymkowiak, Hardie, \& Scott-Brown, 2011) which require the anticipation of events.

It has to be acknowledged that we used a rather simple scenario. Future investigations should consider more complex scenarios containing more than just one dynamic, relevant object. 
Furthermore, the cue classification has to be tested with other traffic scenarios to endorse a generalization of the proposed situational characteristics, which served as anticipatory cues.

\section{REFERENCES}

Bates, D., Mächler, M., Bolker, B., \& Walker, S. (2015). Fitting Linear Mixed-Effects Models Using lme4. Journal of Statistical Software, 67(1), 1-48. https://doi.org/10.18637/jss.v067.i01

Baumann, M., Petzoldt, T., Groenewoud, C., Hogema, J., \& Krems, J. F. (2008). The Effect of Cognitive Tasks on Predicting Events in Traffic. In C. Brusque (Ed.), Proceedings of the European Conference on Human Centred Design for Intelligent Transport Systems (pp. 312). Lyon: HUMANIST Publications.

Deery, H. A. (1999). Hazard and Risk Perception among Young Novice Drivers. Journal of Safety Research, 30(4), 225-236. https://doi.org/10.1016/S0022-4375(99)00018-3

Durso, F. T., Rawson, K. A., \& Girotto, S. (2007). Comprehension and Situation Awareness. In F. T. Durso, R. S. Nickerson, S. T. Dumais, S. Lewandowsky, \& T. J. Perfect (Eds.), Handbook of Applied Cognition (2nd ed., pp. 163-193). Chichester, UK: John Wiley \& Sons Ltd. https://doi.org/10.1002/9780470713181.ch7

Endsley, M. R. (1995). Toward a Theory of Situation Awareness in Dynamic Systems. Human Factors: The Journal of the Human Factors and Ergonomics Society, 37(1), 32-64. https://doi.org/10.1518/001872095779049543

Jackson, L., Chapman, P., \& Crundall, D. (2009). What happens next? Predicting other road users' behaviour as a function of driving experience and processing time. Ergonomics, 52(2), 154-164. https://doi.org/10.1080/00140130802030714

Mühl, K., \& Baumann, M. (2018). The role of cognitive distraction and characteristics of situation elements on anticipation while driving. In Book of Abstracts of the 6th International Conference on Driver Distraction and Inattention. Gothenburg.

Mühl, K., Stoll, T., \& Baumann, M. (2019). Look ahead - understanding cognitive anticipatory processes based on situational characteristics in dynamic traffic situations. Manuscript Submitted for Publication.

Patten, C. J. D., Kircher, A., Östlund, J., Nilsson, L., \& Svenson, O. (2006). Driver experience and cognitive workload in different traffic environments. Accident Analysis \& Prevention, 38(5), 887-894. https://doi.org/10.1016/J.AAP.2006.02.014

Scrimgeour, A., Szymkowiak, A., Hardie, S., \& Scott-Brown, K. (2011). Gender and Hazard Perception Skills in Relation to Road Traffic Police Officers. The Police Journal, 84(4), 333-343. https://doi.org/10.1350/pojo.2011.84.4.524

Stahl, P., Donmez, B., \& Jamieson, G. A. (2014). Anticipation in Driving: The Role of Experience in the Efficacy of Pre-event Conflict Cues. IEEE Transactions on HumanMachine Systems, 44(5), 603-613. https://doi.org/10.1109/THMS.2014.2325558 